\section{CPS-253 THE IMPORTANCE OF THE PHARMACOKINETIC PROFILE IN PATIENTS WITH ULTRA-RARE DISEASES: A CASE REPORT}

C Castillo-Martin*, J Cordero-Ramos, R Castillejo García, A Martínez Suárez, L Rendón De Lope, M Camean Fernández. Hospital Universitario Virgen Macarena, Pharmacy, Seville, Spain

\subsection{6/ejhpharm-2021-eahpconf.85}

Background and importance Mucopolysaccharidosis VII (MPSVII), also known as Sly syndrome, is an ultra-rare disease characterised by deficiency of $\beta$-glucuronidase. Sly phenotypes vary from severe forms with hydrops fetalis and skeletal dysplasia, hepatosplenomegaly, heart valve abnormalities and mental retardation, to milder forms with fewer manifestations.

Aim and objectives To compare the vancomycin pharmacokinetic (PK) profile observed in a newborn with MPSVII with that expected in an average neonate.

Material and methods Clinical data were collected from the electronical medical record (Diraya), and an extensive literature research was made using different electronic databases (Pubmed, Scopus). Serum concentration-time profiles were adjusted to a one compartment neonatal population PK model incorporating body weight and renal function as the significant covariates, using the Abottbase PK System (PKS) programme.

Results The patient was a 26-day-old male, with a postmenstrual age of 38 weeks, and diagnosed with MPS VII, who initially had phlebitis and fever during his stay in the neonatal intensive care unit. His blood cultures were positive for coagulase negative Staphylococcus aureus. The patient was treated with vancomycin $10 \mathrm{mg} / \mathrm{kg} / 8$ hours intravenously. PK were evaluated before the sixth dose, with weight of $2.2 \mathrm{~kg}$, height $44 \mathrm{~cm}$ and a creatinine serum level of $0.92 \mathrm{mg} / \mathrm{L}$. After obtaining a serum level of $123.6 \mu \mathrm{g} / \mathrm{mL}$ (normal trough range 10-15 $\mu \mathrm{g} / \mathrm{mL}$ ), vancomycin was stopped. After 2 days, serum levels were $11.4 \mu \mathrm{g} / \mathrm{mL}$, so vancomycin was restarted at 10 $\mathrm{mg} / \mathrm{kg} / 12$ hours. After four administrations, serum levels were again out of range $(48.2 \mu \mathrm{g} / \mathrm{mL})$, and the antimicrobial was switched to cloxacillin. Based on the vancomycin levels, we estimated a half-life of 15.8 hours, instead of the 4-8 hours described. The distribution volume calculated was $1.99 \mathrm{~L}$ with a clearance of $0.088 \mathrm{~L} /$ hour. The expected distribution volume was $1.8 \mathrm{~L}$ and a clearance of $0.148 \mathrm{~L} /$ hour.

Unfortunately, the baby passed away 3 days later due to other complications.

Conclusion and relevance A 2-3 times greater half-life was observed in this patient with Sly syndrome. The large accumulation of vancomycin was not described in the literature and was not expected with the features of this disease, highlighting the importance of therapeutic drug monitoring in patients with ultra-rare diseases whose pharmacokinetics could be disturbed by factors still unknown.

\section{REFERENCES AND/OR ACKNOWLEDGEMENTS}

Conflict of interest No conflict of interest

\section{CPS-254 A RETROSPECTIVE STUDY OF ANTIMICROBIAL STEWARDSHIP IN A UNIVERSITY HOSPITAL}

${ }^{1} \mathrm{~A}$ De Francesco, ${ }^{1} \mathrm{M}$ Zito, ${ }^{1} \mathrm{~S}$ Esposito, ${ }^{1} \mathrm{C}$ Monopoli, ${ }^{2} \mathrm{MD}$ Naturale, ${ }^{1} \mathrm{M}$ De Fina*. ${ }^{1}$ Azienda Ospedaliera Universitaria 'Mater Domini', Hospital Pharmacy Unit, Catanzaro, Italy; ${ }^{2}$ Magna Graecia University, Hospital Pharmacy Resident, Catanzaro, Italy

10.1136/ejhpharm-2021-eahpconf.86
Background and importance Misuse and abuse of antibiotics are among the main causes of the increase in antibiotic resistance. Monitoring and evaluation of antibiotic prescriptions is an important activity involving the hospital pharmacist.

Aim and objectives The aim of the study was to assess attitudes and practices towards antibiotics. The objectives were to assess clinical governance, prescriptive appropriateness as well as costs incurred.

Material and methods A retrospective observational study was carried out from 1 January 2017 to 31 December 2019 in a university hospital. Outpatient dispensing was used for patient identification and data collection. Demographic, diagnostic, therapeutic and clinical variables were gathered. Consumption was expressed as defined daily dose (DDD). Drugs evaluated were: tigecycline, ceftazidime and beta-lactamase inhibitor, meropenem, ertapenem, ceftaroline, fosamil, ceftolozane and beta-lactamase inhibitor, levofloxacin, dalbavancin, linezolid, daptomycin, amphotericin B, voriconazole, caspofungin, micafungin and anidulafungin. First dispensation date was considered as the index date. Custom requests (CR) that reported prescribing errors were considered inappropriate. Drug costs were calculated based on ex factory prices (VAT excluded), net of the temporary reductions provided for by law. Avoided costs were calculated based on inappropriate prescriptions and unauthorised treatments.

Results 4017 CR, 1267 patients (70.72\% men; mean age 66.54 years) and $26457.22 \mathrm{DDD}$ (19.89 DDD/patient) were included in the study. The expenditure incurred was 1214 $876.87 €$. Data showed a significant decrease in the patient treated rate $(-2 \%)$, DDD required (delta 2019-2017 = $-9.33 \%$ ) and expenditure incurred (delta 2019$17=-52.65 \%)$. The consumption (DDD/pz) of levofloxacin did not increase during the study period (mean 11.22 DDD/ $\mathrm{pz}$ ), while a considerable increase was highlighted for ceftaroline, fosamil and micafungin. Systemic antifungal therapy was started empirically in 181 patients $(68.5 \%$ men; mean age 65 years). Daptomycin was used for persistent methicillin resistant Staphylococcus aureus bacteraemia (delta 2019-2018= $+191.43)$. 3.68\% of CR (148/4017) were deemed inappropriate $(56.4 \%$ in 2019). Costs saved were $29730.37 €$. Prescribed daily dose represented the most common error (20.94\%) in the CP examined.

Conclusion and relevance Hospital pharmacists detected and prevented harmful errors in prescribing therapies. Supervision by hospital pharmacists can significantly improve the management of clinical risk, patient safety, optimisation of care and effective management of expenditure.

\section{REFERENCES AND/OR ACKNOWLEDGEMENTS}

1. WHO Collaborating Centre for Drug Statistics Methodology. ATC/DDD Index 2014. http://www.whocc.no/atc_ddd_index/

Conflict of interest No conflict of interest

\section{CPS-255 CLINICAL EXPERIENCE OF CEFTAROLINE USE IN A THIRD LEVEL HOSPITAL}

A Magallon Martinez*, R Huarte Lacunza, A Pinilla Rello, L Cazorla Poderoso, M Pérez Moreno, A López Pérez, J Perales Pascual, MR Abad Sazatornil. Universitary Miguel Servet Hospital, Hospital Pharmacy, Zaragoza, Spain

10.1136/ejhpharm-2021-eahpconf.87

Background and importance Ceftaroline is approved for the treatment of complicated skin and soft tissue infections 
(cSSSI), and community acquired pneumonia (CAP). However, the most specific aspect of the drug is that it is a beta-lactam with activity against methicillin resistant Staphylococcus aureus (MRSA). These characteristics mean that a large part of its use in clinical practice is in off-label indications.

Aim and objectives To determine the use of ceftaroline in the clinical practice of a third level hospital, and its effectiveness and safety.

Material and methods An observational retrospective study was conducted that included all patients treated with ceftaroline in the hospital from May 2016 to September 2020. Variables studied were: age, sex, indication, dose, microorganism, clinical and microbiological cure, and adverse effects.

Results 57 patients received treatment with ceftaroline, 75.4\% men, with a median age of 69 years (28-89). In 11/57 patients it was used as empirical treatment (for suspected multiresistant germ) and in $46 / 57$ as directed treatment, for the following indications: 13 bacteraemia, 14 endocarditis (with bacteraemia), 15 pneumonia (11 with bacteraemia, 1 with CNS infection and 2 with cSSSI), 2 cSSSI and 2 CNS infection. Infections were caused by MRSA in 23/46 patients, 15/ 46 by methicillin resistant coagulase negative Staphylococcus, 5/46 methicillin sensitive Staphylococcus aureus (MSAS), 2/46 Streptococcus pneumoniae and in 1 patient due to MSAS and Streptococcus pneumoniae. Methicillin resistant microorganisms caused the infections in 38/46 patients. The median duration of treatment was 7 days (1-42). Posology: $600 \mathrm{mg} / 8$ hours was used in bacteraemia, endocarditis and in CNS infection, and $600 \mathrm{mg} / 12$ hours in cSSSI. The regimen used in pneumonia was $600 \mathrm{mg} / 12$ hours in $6 / 15$ patients and $600 \mathrm{mg} / 8$ hours in $9 / 15$ patients ( 8 of whom had bacteraemia and one had concomitant CNS infection). The dosage used in the empirical treatment was $600 \mathrm{mg} / 12$ hours in eight patients and $600 \mathrm{mg} / 8$ hours in three. In six patients it was adjusted for renal function. $78.7 \%$ of the patients presented with clinical resolution $(82.9 \%$ microbiological). Thrombopenia was detected in two patients, probably associated with treatment with the drug.

Conclusion and relevance Our results suggested that ceftaroline was effective in severe cases of methicillin resistant gram positive infections. In most cases, ceftaroline was used for off-label indications. In these cases, higher dosages are being recommended, which are usually prolonged in time, so it is advisable to evaluate the profile of adverse effects.

\section{REFERENCES AND/OR ACKNOWLEDGEMENTS}

Conflict of interest No conflict of interest

\section{CPS-256 HEPATITIS C RETREATMENT OF A PATIENT WHO FAILED MULTIPLE TREATMENTS INCLUDING PROTEASE INHIBITOR AND NON-STRUCTURAL PROTEIN 5A INHIBITORS: A CASE REPORT}

JM Sotoca*, M Rodriguez-Reyes. Hospital Clinic Barcelona, Pharmacy Service, Barcelona, Spain

\subsection{6/ejhpharm-2021-eahpconf.88}

Background and importance It is well documented that direct acting antiviral (DAA) combinations in the treatment of hepatitis $\mathrm{C}$ virus (HCV) achieve high rates of sustained virologic response. However, studies on retreatment options for patients who have failed several DAA treatment regimens that include non-structural protein 5A (NS5A) inhibitors remain scarce.

Aim and objectives To assess the efficacy of glecaprevir/pibrentasvir plus sofosbuvir and ribavirin for 12 weeks in genotype $1 \mathrm{~b}$ patient with compensated cirrhosis who had virologic failure to multiple treatments including regimens containing NS5A inhibitors.

Material and methods A 50-year-old man failed multiple hepatitis C treatments, including peginterferon plus ribavirin (2003) for 9 months, sofosbuvir/ledipasvir plus ribavirin (2014) for 24 weeks, sofosbuvir/simeprevir plus ribavirin (2015) for 24 weeks and sofosbuvir/velpatasvir plus ribavirin (2018) for 24 weeks. Adherence to these treatments was correct according to the dispensing records. Headache, fatigue, anaemia, nausea and pruritus were reported with oral treatments, but drug withdrawal was not required. However, peginterferon was stopped due to anxiety and depression.

There are no specific algorithms to guide retreatment decisions. These must be guided by the drugs administered in previous treatment courses or, if resistance testing is performed, by probabilities of response according to the resistance profile and the treating team's experience. Resistance testing showed resistance to NS5A inhibitors: daclatasvir, elvasvir, ledipasvir, ombitasvir and velpatasvir, but not to pibrentasvir. The multidisciplinary team decided on a new treatment with glecaprevir/pibrentasvir plus sofosbuvir and ribavirin for 12 weeks and it was started in November 2019. Prior to treatment, drug interactions were checked. An undetectable HCV RNA level 12 weeks after completion of therapy (SVR12) defined treatment success.

Results Two potential drug interactions were detected: (1) gemfibrozil was discontinued because of the increased risk of gastrointestinal side effects; (2) due to the potential increase in carvedilol concentrations, close monitoring of heart rate and blood pressure was recommended. The patient achieved SVR12 with the fifth hepatitis C treatment with glecaprevir/ pibrentasvir plus sofosbuvir and ribavirin for 12 weeks. The treatment was well tolerated, and adherence was correct.

Conclusion and relevance In this particularly difficult to cure cirrhotic patient previously exposed to NS5A inhibitors, the combination of glecaprevir/pibrentasvir plus sofosbuvir and ribavirin administered for 12 weeks achieved SVR12.

\section{REFERENCES AND/OR ACKNOWLEDGEMENTS}

Conflict of interest No conflict of interest

\section{CPS-257 RELEVANCE OF RITONAVIR INTERACTIONS IN HIV TREATMENTS THAT INVOLVE TREATMENT MODIFICATION}

I Beltrán García*, MJ Cuellar-Monreal, M Centelles-Oria, T Palanques-Pastor, E MonteBoquet, A García-Robles, MV Tarazona-Casany, JL Poveda-Andrés, R Iglesias-Gomez, O Ballesta-López, A Solana-Altabella. Sefh, Hospital Universitario Y Politécnico La Fe, Valencia, Spain

\subsection{6/ejhpharm-2021-eahpconf.89}

Background and importance The protease inhibitor (PI)/ enhancer combination, more than 20 years after its appearance, continues to be the antiretroviral therapy (ART) of choice in certain circumstances due to its high genetic barrier. Ritonavir (RTV) acts as an enhancer and has a higher potential for drug interactions. 